\title{
Universiteit
}

Leiden

The Netherlands

\section{Altered connectivity between prefrontal and sensorimotor cortex in conversion paralysis.}

Lange, F.P. de; Toni, I.; Roelofs, K.

\section{Citation}

Lange, F. P. de, Toni, I., \& Roelofs, K. (2010). Altered connectivity between prefrontal and sensorimotor cortex in conversion paralysis. Neuropsychologia, 48, 1782-1788. Retrieved from https://hdl.handle.net/1887/15723

Version: $\quad$ Not Applicable (or Unknown)

License: $\quad$ Leiden University Non-exclusive license

Downloaded from: https://hdl.handle.net/1887/15723

Note: To cite this publication please use the final published version (if applicable). 


\title{
Altered connectivity between prefrontal and sensorimotor cortex in conversion paralysis
}

\author{
Floris P. de Lange ${ }^{\mathrm{a}, *}$ Ivan Toni $^{\mathrm{a}}$, Karin Roelofs ${ }^{\mathrm{b}, \mathrm{c}}$ \\ a Donders Institute for Brain, Cognition and Behavior, Radboud University Nijmegen, Kapittelweg 29, 6500 HB Nijmegen, Netherlands \\ ${ }^{\mathrm{b}}$ Leiden University-Institute of Psychology, Clinical, Health and Neuropsychology Unit, Netherlands \\ ${ }^{c}$ Leiden Institute for Brain and Cognition (LIBC), Netherlands
}

\section{A R T I C L E I N F O}

\section{Article history:}

Received 20 October 2009

Received in revised form 17 February 2010

Accepted 25 February 2010

Available online 4 March 2010

\section{Keywords:}

fMRI

Mental rotation

Motor imagery

Conversion disorder

Dorsolateral prefrontal cortex

Connectivity

\begin{abstract}
A B S T R A C T
Conversion paralysis (CP) is a frequent and impairing psychiatric disorder, affecting voluntary motor function. Yet, we have previously shown that the motor system of $\mathrm{CP}$ patients with a unilateral conversion paresis is recruited to a similar degree during imagined movements of the affected and unaffected limb. In contrast, imagery of movements with the affected limb results in larger prefrontal activation. It remains unclear how this hand-specific increased prefrontal activity relates to the reduced responsiveness of motor and somatosensory areas, a consistent and important feature of $\mathrm{CP}$ patients.

In the current study, we investigated changes in the inter-regional coupling between prefrontal cortex (PFC) and sensorimotor regions when CP patients imagined movements involving either the affected or the unaffected hand. We found that there were distinct connectivity patterns for different parts of the PFC. While ventromedial PFC was not functionally connected to the motor system, we observed strong functional coupling between the dorsolateral PFC and various sensorimotor areas. Furthermore, this coupling was modulated by whether patients imagined movements of their affected or unaffected hand. Together, these results suggest that the reduced motor responsitivity observed in CP may be linked to altered dorsolateral prefrontal-motor connectivity.
\end{abstract}

(c) 2010 Elsevier Ltd. All rights reserved.

\section{Introduction}

Conversion paralysis (CP) is a psychiatric disorder that is characterized by a loss of voluntary motor functioning. Although the symptoms may suggest a neuropathological condition, they cannot be adequately explained by known neurological or other organic disorders (American Psychiatric Association, 1994). Moreover, the onset or exacerbation of symptoms is related to psychological stress, suggesting that psychological mechanisms play an important role (Roelofs \& Spinhoven, 2007). Despite the high prevalence of $\mathrm{CP}$ and the long history of speculations about its cause (Halligan, Bass, \& Marshall, 2001; Vuilleumier, 2005), the exact nature of CP remains poorly understood.

Several neuroimaging studies have tried to explore the objective neural correlates of functional mechanisms that, in the absence of a structural brain lesion, may be involved in CP. These studies have generally implicated ventromedial (Halligan, Athwal, Oakley, \& Frackowiak, 2000; Marshall, Halligan, Fink, Wade, \& Frackowiak, 1997) and dorsolateral (Spence, Crimlisk, Cope, Ron, \& Grasby,

\footnotetext{
* Corresponding author. Tel.: +31 2436 10658; fax: +31 243610652

E-mail address: floris.delange@donders.ru.nl (F.P. de Lange).
}

2000) regions within the prefrontal cortex during (failed) attempts of movements. Given the role of these regions in the internal generation of actions (Fuster, 2000; Passingham, 1993), these findings were interpreted as reflecting active motor inhibition.

In most of these studies however, patients were explicitly asked to attempt to move their paralyzed limb, which may also trigger emotional and motivational responses, thus complicating the interpretation of the results (Price \& Friston, 2002). To overcome the interpretational limitations imposed by overt motor behavior, some studies have studied cerebral responses evoked by passive sensory stimulation (Vuilleumier et al., 2001) or action observation (Burgmer et al., 2006). Vuilleumier et al. assessed brain responsiveness to passive sensory stimulation in $\mathrm{CP}$ patients suffering from unilateral sensorimotor loss in a single photon emission computerized tomography (SPECT) study. The results showed decreased activity in the basal ganglia and thalamus contralateral to the affected limb during stimulation of the affected limb compared to the unaffected limb. This decrease resolved after recovery of the conversion symptoms, suggesting that $\mathrm{CP}$ is associated with altered somatosensory processing. Burgmer et al. (2006) explored whether CP is associated with abnormal brain activity during observation of hand movements. The authors showed that compared to healthy controls, CP patients had reduced primary motor cortical 
activity during observation of hand movements, specifically for the affected hand. Although these findings do not suffer from the interpretational problems related to overt motor execution, it remains to be determined how these sensory and action observation deficits relate to the main feature of $\mathrm{CP}$, namely the disturbance of volitional motor processes.

A well-established approach to directly probe the motor system in absence of overt motor behavior makes use of motor imagery. This approach controls for neural processes associated with actual motor execution like altered sensory feedback or enhanced monitoring of failed movements. This approach to study the generation and preparation of actions is supported by a wealth of evidence showing that imagined and executed movements overlap in terms of time course (Parsons, 1987; Sekiyama, 1982), autonomic responses (Decety, Jeannerod, Durozard, \& Baverel, 1993), and neural architecture (de Lange, Hagoort, \& Toni, 2005; Jeannerod, 1994; Parsons, Gabrieli, Phelps, \& Gazzaniga, 1998). Indeed, previous behavioral studies have succesfully used motor imagery tasks to reveal impairments in motoric simulations of the affected limb in patients with CP (Maruff \& Velakoulis, 2000; Roelofs et al., 2001). Two imaging experiments using motor imagery in CP patients with a lateralized paresis extended these behavioral findings. The first study showed that while preparatory motor structures were activated similarly during motor imagery for the affected and the unaffected limb, there was larger activation in several prefrontal clusters during motor imagery of the affected limb (de Lange, Roelofs, \& Toni, 2007). Given the role of these regions in selfreflexive processing (Goldberg, Harel, \& Malach, 2006) as well as observation and awareness of actions (Castelli, Happe, Frith, \& Frith, 2000; Frith, Blakemore, \& Wolpert, 2000a), we speculated that these activation differences were due to heightened selfmonitoring of (imagined) actions with the affected arm. In a second study, we found that these activation differences disappeared when subjects were explicitly instructed to focus on the "to be imagined" movements (de Lange, Roelofs, \& Toni, 2008), lending further support to the idea that increased self-monitoring of actions underlies the increased activation for imagery of actions with the affected arm.

However, it remains unclear how the increased prefrontal responses relate to another consistent feature of CP patients, namely reduced responsiveness of motor and somatosensory areas. For instance, a recent study that directly probed the excitability of the motor cortex using transcranial magnetic stimulation observed striking excitability changes in the motor system during motor imagery of the affected hand in CP (Liepert, Hassa, Tüscher, \& Schmidt, 2009). While motor imagery of the unaffected hand resulted in higher motor excitability, as it is normally observed in the healthy population (Abbruzzese, Trompetto, \& Schieppati, 1996), motor imagery of the affected hand led to a paradoxical decrease of motor excitability. These results are in accord with other studies showing reduced responses in motor (Burgmer et al., 2006; Kanaan, Craig, Wessely, \& David, 2007; Stone et al., 2007) and somatosensory areas (Ghaffar, Staines, \& Feinstein, 2006; MailisGagnon et al., 2003) in CP.

In this study, we used connectivity analyses to assess the relationship between local responses evoked in prefrontal and sensorimotor cortex during a motor imagery task involving either the affected or non-affected hand in CP (de Lange et al., 2007). We observed strong functional coupling between the dorsolateral PFC and various sensory and motor areas. Crucially, the strength of this coupling was modulated by whether patients imagined movements of their affected or unaffected hand. These results suggest that the reduced responsiveness of sensorimotor responses, previously observed in CP patients, could arise from altered connectivity between dorsolateral prefrontal and sensorimotor regions.

\section{Materials and methods}

\subsection{Participants}

We studied eight patients (mean age of 34.6 years, range $18-56, \mathrm{SD}=13.2$ ) diagnosed with conversion disorder according to the DSM-IV criteria (American Psychiatric Association, 1994) and showing a full or partial paralysis lateralized to one arm as a major symptom. For a full description of inclusion criteria and diagnosis procedure, see de Lange et al. (2007). The study was approved by the local medical ethical committee and all patients gave their informed consent before participation.

\subsection{Task}

We used a well-known motor imagery task, in which the participants have to judge the laterality of the visually presented rotated hand stimulus (Parsons, 1987). We used line drawings of left and right hands, in different orientations varying from $0^{\circ}$ to $180^{\circ}$ in $45^{\circ}$ steps. The hand could be shown in either palmar or dorsal orientation. The stimuli were serially presented to the patients in a random order. For each trial, the hand stimulus was presented centrally on the screen, and patients were instructed to judge as fast and as accurately as possible whether the stimulus constituted a left or a right hand. When the patient provided his/her response, the stimulus was replaced with a fixation cross, which stayed on until the start of the next trial (inter-trial interval: $1.5-2.5 \mathrm{~s}$ ). The experiment consisted of 160 trials of motor imagery. After a series of 10 motor imagery trials, a rest period of $10 \mathrm{~s}$ was introduced to sample baseline activity. During this rest period, patients were instructed to look at the fixation cross.

Patients responded by pressing one of two buttons attached to their left and right big toe. The patients' left and right feet were firmly attached to a button box, and reaction times and error rates were measured for subsequent behavioral analysis. The stimuli were presented using Presentation software (Neurobehavioral systems, Albany, USA), and they were projected onto a screen at the back of the scanner and seen through a mirror above the patients' heads. The main effects of hand (affected vs. unaffected) and stimulus rotation on reaction times and cerebral activity have been described in a previous study on the same data set (de Lange et al., 2007). Here, we focus on functional and effective connectivity as a function of motor imagery of the affected vs. the unaffected hand, using the previously described prefrontal clusters as target seed regions.

\subsection{MRI acquisition and preprocessing}

Functional images were acquired on a Siemens (Erlangen, Germany) $1.5 \mathrm{~T}$ MRI system equipped with echo planar imaging (EPI) capabilities using the standard head coil for radio frequency transmission and signal reception. Functional images were acquired using a gradient EPI-sequence (TE/TR $=40 / 2540 \mathrm{~ms} ; 32$ axial slices, voxel size $=3.5 \mathrm{~mm}$; FOV $=224 \mathrm{~mm}$ ). On average, the duration of the experiment was 23 min in which 547 scans were acquired. High-resolution anatomical images were acquired using a MP-RAGE sequence (TE/TR $=3.93 / 2250 \mathrm{~ms}$; voxel size $=1.0 \mathrm{~mm}, 176$ sagittal slices; FOV $=256 \mathrm{~mm}$ ). Preprocessing of the functional data and calculation of the contrast images for statistical analysis was done with SPM5 (www.fil.ion.ucl.ac.uk/spm). First, functional images were realigned, slice-time corrected, normalized to a common stereotactic space (MNI: Montreal Neurological Institute, Canada) and smoothed with a $10 \mathrm{~mm}$ FWHM Gaussian kernel. Our spatial filter size was motivated by both the sizable inter-individual differences in prefrontal anatomy (Rajkowska \& Goldman-Rakic, 1995) and in order to optimize sensitivity in the small sample of patients used (Petersson, Nichols, Poline, \& Holmes, 1999). By jittering trial onsets with respect to image acquisition and randomizing stimulus rotations, our experimental design allowed for an event-related analysis of the fMRI time series.

\subsection{Functional and effective connectivity analysis}

We carried out an analysis of functional and effective connectivity, using the psychophysiological interaction (PPI) method to test for (changes in) connectivity between our seed regions and other brain areas (Friston et al., 1997). Functional connectivity ensues from significantly correlated activity patterns over time between separate regions. Effective connectivity, on the other hand, ensues from significant changes in correlation strength between separate regions as a function of task context. We placed seed regions in previously described clusters in the prefrontal cortex (PFC), namely the ventromedial PFC (vmPFC: $[8,44,-24]$; putative BA 11), dorsomedial PFC (dmPFC: [-12,62,32]; putative BA 10) and dorsolateral PFC (dIPFC: [-36,48,34]; putative BA 46/9). Each of these regions was defined by the first eigenvariate of the time series of all voxels within a $6 \mathrm{~mm}$ sphere surrounding the peak activations. These regions were chosen as they constituted the local maxima of differential activation between motor imagery of the affected vs. the unaffected hand (see de Lange et al., 2007). The PPI method makes inferences about regionally specific responses caused by the interaction between an experimentally manipulated psychological factor and the physiological activity measured in a given index area. The analysis was constructed to test for differences in the regression slope of PFC activity on other brain areas, depending on whether subjects performed motor imagery of the affected vs. the unaffected hand. To construct our PPI regressor we used a hemo- 
dynamic deconvolution method (Gitelman, Penny, Ashburner, \& Friston, 2003). This PPI regressor, alongside the task regressors and the time course of the PFC seed region, were included in the statistical model. To remove any artifactual signal changes due to head motion, we also included 6 parameters describing the headmovements (three translations, three rotations) as confounds in the model. Linear contrasts pertaining to the main effects of the factorial design constituted the data for the second-stage analysis, which treated participants as a random factor. In this second-stage analysis, we carried out two analyses: (1) analysis of functional connectivity with the PFC (as parameterized by the regressor describing the time course of the PFC seed region) and (2) analysis of effective connectivity with the PFC as a function of affected vs. unaffected hand (as parameterized by the PPI regressor).

We report the results of random effects analyses, using the family-wise error (FWE) correction method to control the false alarm rate (Friston et al., 1995). For our analysis of functional connectivity, we used a relatively stringent voxel-based statistical threshold (voxel threshold: $p_{\mathrm{FWE}}=0.05$ ) and cluster threshold $(k>10)$, in order to limit spurious correlations that may be the result of general co-fluctuations during the scanning session. For analyses of effective connectivity, we used the more sensitive cluster-based correction for multiple comparisons (Friston, Holmes, Poline, Price, \& Frith, 1996). For these analyses, we corrected for multiple comparisons at the cluster level $(p<0.05)$, based on a voxel level threshold of $p<0.01$. We also tested for effective connectivity changes within a predefined set of regions that has previously been identified to be related to motor imagery, comprising the bilateral intraparietal sulcus (IPS) and dorsal premotor cortex (PMd). Within this limited search space, we corrected for multiple comparisons within $6 \mathrm{~mm}$ spheres surrounding previously identified peak maxima (see de Lange et al., 2005; de Lange, Helmich, \& Toni, 2006; de Lange et al., 2007).

\subsection{Anatomical inference}

Anatomical details of significant signal changes were obtained by superimposing the relevant SPMs on the structural images of the subjects. The atlas of Duvernoy, Cabanis, and Vannson (1991) was used to identify relevant anatomical landmarks. When applicable, Brodmann areas (BAs) were assigned on the basis of the SPM Anatomy Toolbox (Eickhoff et al., 2005).

\section{Results}

We carried out analyses of functional and effective connectivity in three regions within the prefrontal cortex that were more active during motor imagery of affected than unaffected hands: ventromedial (vmPFC), dorsomedial (dmPFC) and dorsolateral (dlPFC) prefrontal cortex (Amodio \& Frith, 2006). vmPFC was strongly functionally connected to the left $([-60,-10,-32] ; T=23.1$, $\left.p_{\mathrm{FWE}}=0.007\right)$ and right $\left([58,-14,-30] ; T=19.9, \quad p_{\mathrm{FWE}}=0.021\right)$ inferior temporal cortex. dmPFC showed significant functional connectivity with its contralateral counterpart ([18,60,34]; $T=38.2$, $\left.p_{\text {FWE }}<0.001\right)$. These vmPFC and dmPFC clusters did not show significant coupling with any of the nodes of the sensorimotor network. Importantly, these clusters also did not show any significant changes in effective connectivity as a function of hand laterality.
In contrast, dIPFC showed strong functional coupling with several nodes of the sensorimotor system (Fig. 1). This analysis identified a network of regions that comprised the supramarginal gyrus [assigned to parietal area PF with $70 \%$ probability (Caspers et al., 2006; Eickhoff et al., 2005)], supplementary motor area and the central sulcus, encompassing both the precentral gyrus (assigned to area $3 \mathrm{~b}$ with $60 \%$ probability) and postcentral gyrus [assigned to area 1 with 70\% probability (Geyer, Schleicher, \& Zilles, 1999)]. The precise anatomical localization of these clusters is provided in Table 1 . The dense functional coupling between the dIPFC region and these sensorimotor regions, which are all involved in aspects of generation and planning of motor commands, is in good correspondence with the hypothesized role of the dIPFC seed region in the selection of action plans (Frith, Moody, \& Driver, 2000b). We next investigated which regions showed stronger positive or negative coupling with the dIPFC as a function of whether subjects imagined movements of the affected vs. the unaffected hand.

\subsection{Increased positive coupling between dIPFC and dorsal premotor cortex}

We observed larger positive coupling for motor imagery of the affected hand compared to the unaffected hand between the dIPFC and the right dorsal premotor cortex (PMd). Although larger coupling could also be seen between dIPFC and the left PMd $\left([-18,8,64] ; T=4.33, p_{\text {uncorrected }}=0.002\right)$, this result failed to reach significance when correcting for multiple comparisons within the predefined search space. Both left and right PMd showed overall significant increases in cerebral activity with increasing biomechanical complexity (left PMd: $T=8.94, p_{\mathrm{FWE}}=0.0078$; right PMd: $T=7.07, p_{\text {FWE }}=0.0034$ )

There were no other brain regions that showed significantly larger positive coupling for motor imagery of the affected hand compared to the unaffected hand.

\subsection{Increased negative coupling between dlPFC and primary somatosensory cortex and hippocampus}

There were several regions that showed a significantly more negative regression slope as a function of dlPFC activity during motor imagery of the affected hand, compared to the unaffected hand. There was increased negative coupling in the left and right postcentral gyrus [spanning both BA 2 with 40\% probability and BA 3a with $60 \%$ probability (Grefkes, Geyer, Schormann, Roland, \& Zilles, 2001), and the anterior part of the superior parietal lobule].

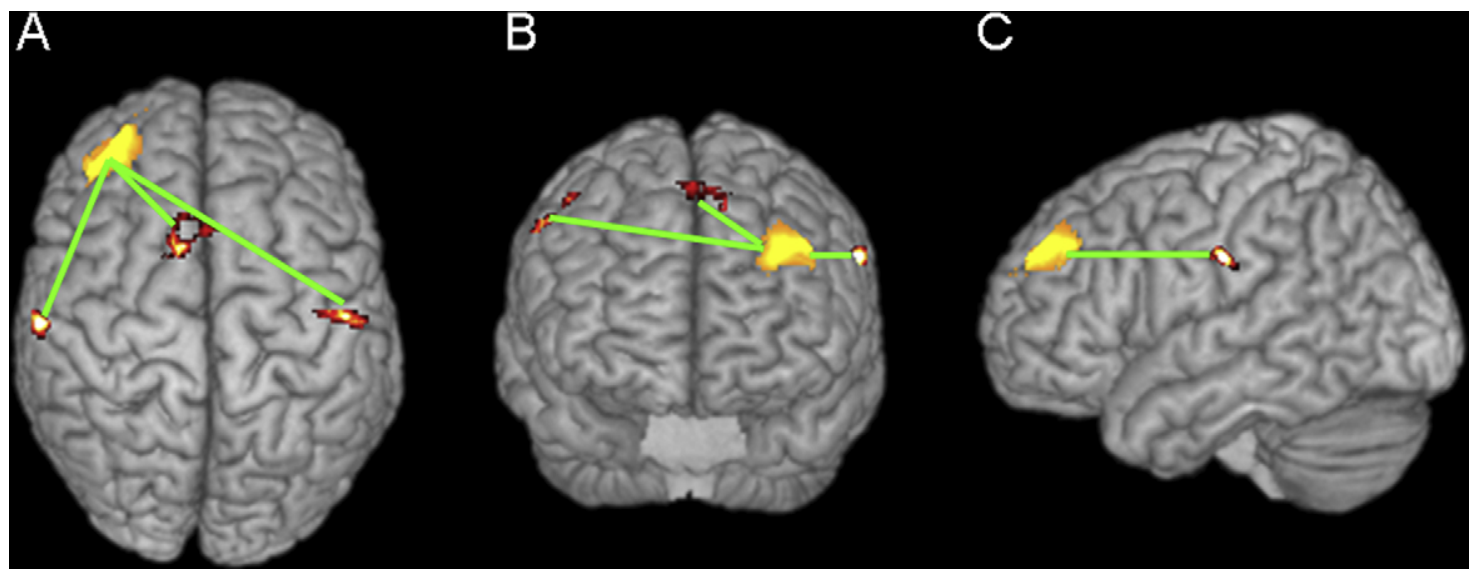

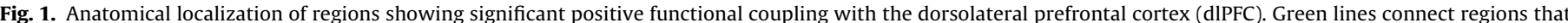

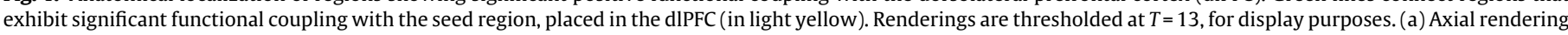

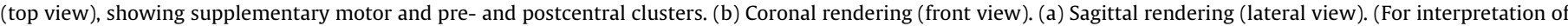
the references to color in this figure legend, the reader is referred to the web version of the article.) 
Table 1

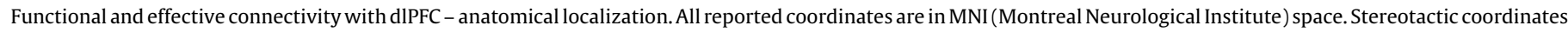
denote the peak of the clusters surviving correction for multiple comparisons.

\begin{tabular}{|c|c|c|c|c|c|c|c|}
\hline \multirow[t]{2}{*}{ Contrast } & \multirow[t]{2}{*}{ Region } & \multirow[t]{2}{*}{$T$-value } & \multirow[t]{2}{*}{ Cluster size } & \multirow[t]{2}{*}{ Corrected $p$-value } & \multicolumn{3}{|c|}{ Stereotactic coordinates } \\
\hline & & & & & $x$ & $y$ & $z$ \\
\hline \multirow[t]{4}{*}{ Functional coupling } & Supplementary motor area & 78.1 & 46 & $<0.001$ & -10 & 8 & 48 \\
\hline & Supramarginal gyrus & 40.3 & 32 & $<0.001$ & -62 & -18 & 28 \\
\hline & & 24.0 & 12 & 0.006 & -58 & -30 & 34 \\
\hline & Central sulcus & 31.5 & 16 & 0.001 & 52 & -16 & 42 \\
\hline Increased positive coupling & Dorsal premotor cortex & 5.2 & 23 & $0.038^{\mathrm{a}}$ & 24 & 2 & 70 \\
\hline \multirow[t]{3}{*}{ Increased negative coupling } & Postcentral gyrus & 16.5 & 343 & 0.002 & 18 & -42 & 52 \\
\hline & & 6.9 & 239 & 0.022 & -18 & -38 & 50 \\
\hline & Hippocampus & 8.4 & 52 & 0.014 & 16 & -16 & -16 \\
\hline
\end{tabular}

a Corrected for multiple comparisons in an a priori defined search space.

There was also increased negative coupling between dlPFC and the right hippocampus.

Activity in the postcentral clusters was overall suppressed during motor imagery (left postcentral gyrus: $T=6.27, p_{\mathrm{FWE}}=0.035$; right postcentral gyrus: $T=9.72, p_{\mathrm{FWE}}<0.001$ ), in line with previous studies (Cheron \& Borenstein, 1992; Jahn et al., 2004). Therefore, the current set of results suggest that activity in dIPFC and suppression of postcentral regions are coupled more strongly during imagery of the affected hand.

\section{Discussion}

Previously, we investigated cerebral activity in 8 patients with a unilateral conversion paralysis during motor simulation of actions with their affected and unaffected hand. While motor imagery recruited comparable cerebral resources in the motor system, CP patients recruited additional cerebral resources in several clusters of the prefrontal cortex during motor imagery of the affected hand (de Lange et al., 2007). In the current study, we build on these findings and examined the functional and effective connectivity profile of the prefrontal cortex during motor imagery. We observed that different clusters within the prefrontal cortex had distinct functional connectivity patterns. While medial prefrontal clusters were functionally connected to inferior temporal regions, only the dorsolateral prefrontal cortex (dIPFC) was functionally connected to the motor system. Moreover, the coupling between this region and various parts of the sensorimotor system was modulated by whether subjects imagined movements of the affected or the unaffected limb: dlPFC showed a stronger positive coupling with the dorsal premotor cortex (PMd), and a stronger negative coupling with the primary somatosensory cortex (S1). Below we will detail and interpret these findings.

\section{1. dIPFC is functionally coupled with the sensorimotor system}

In our previous study, we found differential responses during imagery of the affected and non-affected hand in a large prefrontal cluster, spanning both ventromedial and dorsolateral aspects of the superior frontal gyrus. In other words, this cluster incorporated responses from anatomically distinct regions (Cavada, Company, Tejedor, Cruz-Rizzolo, \& Reinoso-Suarez, 2000; Chiavaras \& Petrides, 2000; Petrides, 2005). Here we separately assessed the functional connectivity of each of the three local maxima of this large cluster. The maxima were localized in ventromedial (vmPFC), dorsomedial (dmPFC) and dorsolateral (dlPFC) prefrontal cortex, close to the location of functional differences reported in previous neuroimaging studies on CP patients (Cojan, Waber, Carruzzo, \& Vuilleumier, 2009; Halligan et al., 2000; Marshall et al., 1997; Spence et al., 2000). The dlPFC showed distinct functional connectivity patterns from the medial clusters. Notably, the dlPFC exhibited significant co-fluctuations with parietal, primary somatosensory, motor and supplementary motor areas (Fig. 1). In contrast, vmPFC and dmPFC clusters were not connected to the motor system, but instead to inferior temporal and contralateral dmPFC clusters. Taken together, this pattern of functional connectivity suggests that although these PFC clusters exibit similar local responses during motor imagery of the affected hand, they differ in terms of the long-range couplings to these local responses. More precisely, the dIPFC is coupled with parts of the motor system, in line with its presumed role in the selection of action plans (Frith et al., 2000b; Passingham, 1993; Rowe et al., 2007) (Fig. 2).

\subsection{Increased positive coupling between dIPFC and PMd during imagery of the affected hand}

Analysis of effective connectivity showed a larger positive coupling between dIPFC and PMd during motor imagery of the affected hand. This means that a unitary increase in dIPFC activity when CP patients were imagining movements of the affected hand resulted in significantly greater activity in the PMd, as compared to trials where the patients imagined movements of the non-affected hand. PMd is one of the two core regions that are specifically involved in mental simulation of hand movements (de Lange et al., 2005). The activity of this region is likely to be related to the generation of motor plans. For example, prolonged electrical stimulation of the macaque's precentral gyrus evokes complex upper-limb movements (Graziano, Taylor, \& Moore, 2002), and premotor neurons are known to select and encode various movement parameters. The increased coupling between dIPFC and PMd during motor imagery of the affected hand may be a cerebral counterpart of the increased attention to action that $\mathrm{CP}$ patients deploy during the generation of action plans of the paralyzed limb (Rowe, Friston, Frackowiak, \& Passingham, 2002). Given the matched motor imagery performance of these CP patients during trials involving either hand (de Lange et al., 2007), it is conceivable that the increase in dlPFC-premotor connectivity reflects a compensatory mechanism, namely an increased prefrontal drive towards premotor regions supporting the imagery process. Future studies might be able to test the directionality of the change in prefrontal-premotor connectivity, as predicted by this hypothesis.

\subsection{Increased negative coupling between dIPFC and S1 during imagery of the affected hand}

The dlPFC had larger negative couplings with the left and right postcentral gyrus, as well as with the hippocampus. The postcentral clusters fell within cyto-architectonic boundaries of primary somatosensory cortices (S1). We interpret our findings as stronger negative coupling during imagery of the affected hand, rather than stronger positive coupling during imagery of the unaffected hand, 


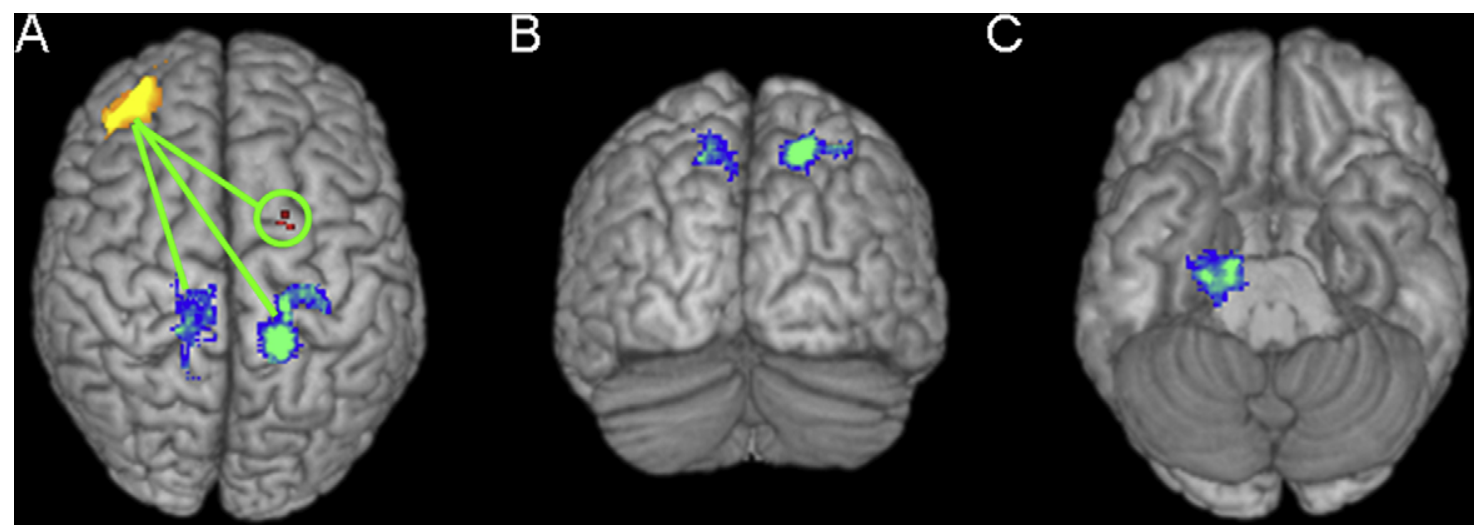

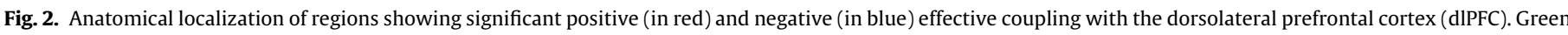

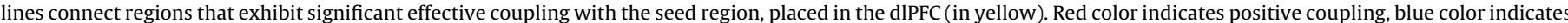

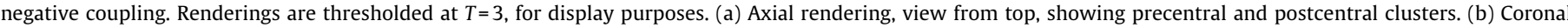

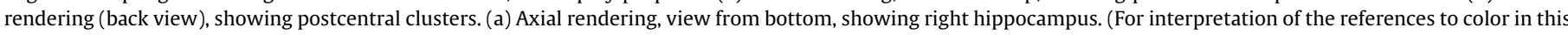
figure legend, the reader is referred to the web version of the article.)

in view of S1 showing strong task-related suppression of activity. Suppression of somatosensory cortex during motor imagery has been previously observed (Jahn et al., 2004; Rossini et al., 1996). Current models of motor control assert that during selfproduced actions, a prediction of the sensory consequences of the action is created, which is subtracted from the observed sensory afferent information (Shadmehr \& Krakauer, 2008; Wolpert \& Ghahramani, 2000). This results in attenuated somatosensory perception, even when motor plans are generated but not executed (Voss, Ingram, Wolpert, \& Haggard, 2008) or merely imagined (Cheron \& Borenstein, 1992; Grush, 2004). The stronger negative coupling between dIPFC and S1 may therefore be directly related to the stronger positive coupling between dIPFC and PMd. When motor plans are generated (in PMd), sensory consequences are simultaneously computed, leading to sensory attenuation (in S1). In line with this, we observed a stronger modulatory influence of dIPFC during the generation of action plans of the affected limb that was positive in sign for the PMd and negative in sign for S1. The stronger inhibitory coupling between dIPFC and S1 during motor imagery of the affected hand could also provide an explanation for the decreased excitability of the primary motor cortex that has previously been observed in CP (Liepert et al., 2009). Activity in S1 can strongly contribute to the excitability of the motor system as measured by motor evoked potentials (Avenanti, Bolognini, Maravita, \& Aglioti, 2007). Our results suggest that this reduced excitability may be the result of larger dIPFC activity during generation of action plans of the affected hand.

\subsection{Coupling between dIPFC and the hippocampus}

We also observed larger negative coupling between dlPFC and the right hippocampus during motor imagery of the affected hand. The hippocampus is known to directly interact with the prefrontal cortex during the formation and retrieval of associative memory traces (Dolan \& Fletcher, 1997; Gaffan, 2005; Schacter \& Wagner, 1999). More specifically, the control of unwanted memories is associated with increased dIPFC activation and reduced hippocampal activation (Anderson et al., 2004). We speculate that the negative coupling between dIPFC and hippocampus observed during imagery of movements involving the affected hand could mediate a control mechanism to decouple (imagined) movements of the affected hand from aversive memories associated with those movements. It remains to be seen whether remission of CP symp- toms is supported by increased efficiency of this putative control mechanism.

\section{5. vmPFC and dlPFC: self-monitoring and action selection?}

While previous studies have often interpreted changes in the prefrontal activation profile of $\mathrm{CP}$ in a unitary fashion, our current set of results, alongside anatomical and functional considerations, suggest that alterations in different nodes of the prefrontal cortex may be linked to distinct aspects of this psychopathological condition. While vmPFC showed functional connectivity with inferior temporal cortices, there was no functional or effective connectivity with the sensorimotor system. The vmPFC has close connections with limbic areas and it is involved in emotion regulation (Ochsner \& Gross, 2005) as well as interoceptive attention and self-referential and autobiographical processing (Amodio \& Frith, 2006; Gilbert et al., 2006; Summerfield, Hassabis, \& Maguire, 2009). Accordingly, we have previously attributed the vmPFC activity differences to heightened self-monitoring during motor imagery of the affected limb. The current set of results extend these observations by showing that vmPFC does not directly impinge on the sensorimotor system.

Conversely, the dlPFC showed extensive functional connectivity with the sensorimotor system, and altered its connectivity as a function of whether $\mathrm{CP}$ patients engaged in motor imagery of the affected or unaffected hand. Therefore, dIPFC may be more directly involved in mediating the altered sensory and motor symptoms observed in CP. It might be relevant to test whether experimental manipulation of dIPFC activity (as achievable with transcranial magnetic stimulation, for instance) could lead to a modulation of the sensorimotor symptoms of CP patients. Therefore, we propose that both mechanisms (heightened self-monitoring and action selection) have a role in conversion paralysis, but subserved by anatomically distinct parts of the prefrontal cortex.

\section{Conclusion}

Our results indicate that there are altered connectivity patterns between the dIPFC and various sensorimotor nodes during the formation of action plans of the affected arm. There is increased positive coupling between dIPFC and PMd, while there is increased negative coupling between dIPFC and S1 and the hippocampus. These results provide a link between previous reports of both heightened prefrontal and reduced sensorimotor activity in $\mathrm{CP}$. 
These results suggest that reduced motor responsitivity may be linked to altered dorsolateral prefrontal-motor connectivity.

\section{Competing interests}

The authors have no competing interests.

\section{Acknowledgments}

This study was supported by the Netherlands Science Foundation (NWO: grant number 446-07-003 awarded to FdL; 452-03-339 awarded to IT and 451-02-115 awarded to KR) and the Dutch Brain Foundation (Hersenstichting Nederland, grant number 12F04(2).19 awarded to KR and FdL).

\section{References}

Abbruzzese, G., Trompetto, C., \& Schieppati, M. (1996). The excitability of the human motor cortex increases during execution and mental imagination of sequential but not repetitive finger movements. Experimental Brain Research, 111(3), 465-472.

American Psychiatric Association. (1994). Diagnostic and statistical manual of mental disorders DSM-IV. American Psychiatric Association.

Amodio, D. M., \& Frith, C. D. (2006). Meeting of minds: The medial frontal cortex and social cognition. Nature Reviews Neuroscience, 7(4), 268-277.

Anderson, M. C., Ochsner, K. N., Kuhl, B., Cooper, J., Robertson, E., Gabrieli, S. W., et al. (2004). Neural systems underlying the suppression of unwanted memories. Science, 303(5655), 232-235.

Avenanti, A., Bolognini, N., Maravita, A., \& Aglioti, S. M. (2007). Somatic and motor components of action simulation. Current Biology, 17(24), 2129-2135.

Burgmer, M., Konrad, C., Jansen, A., Kugel, H., Sommer, J., Heindel, W., et al. (2006) Abnormal brain activation during movement observation in patients with conversion paralysis. Neuroimage, 29(4), 1336-1343.

Caspers, S., Geyer, S., Schleicher, A., Mohlberg, H., Amunts, K., \& Zilles, K. (2006). The human inferior parietal cortex: Cytoarchitectonic parcellation and interindividual variability. Neuroimage, 33(2), 430-448.

Castelli, F., Happe, F., Frith, U., \& Frith, C. (2000). Movement and mind: A functional imaging study of perception and interpretation of complex intentional movement patterns. Neuroimage, 12(3), 314-325.

Cavada, C., Company, T., Tejedor, J., Cruz-Rizzolo, R. J., \& Reinoso-Suarez, F. (2000). The anatomical connections of the macaque monkey orbitofrontal cortex. A review. Cerebral Cortex, 10(3), 220-242.

Cheron, G., \& Borenstein, S. (1992). Mental movement simulation affects the N30 frontal component of the somatosensory evoked potential. Electroencephalography Clinical Neurophysiology, 84(3), 288-292.

Chiavaras, M. M., \& Petrides, M. (2000). Orbitofrontal sulci of the human and macaque monkey brain. Journal of Comparative Neurology, 422(1), 35-54.

Cojan, Y., Waber, L., Carruzzo, A., \& Vuilleumier, P. (2009). Motor inhibition in hysterical conversion paralysis. NeuroImage,

de Lange, F. P., Hagoort, P., \& Toni, I. (2005). Neural topography and content of movement representations. The Journal of Cognitive Neuroscience, 17(1), 97-112.

de Lange, F. P., Helmich, R. C., \& Toni, I. (2006). Posture influences motor imagery: An fMRI study. Neuroimage, 33(2), 609-617.

de Lange, F. P., Roelofs, K., \& Toni, I. (2007). Increased self-monitoring during imagined movements in conversion paralysis. Neuropsychologia, 45(9), 2051-2058.

de Lange, F. P., Roelofs, K., \& Toni, I. (2008). Motor imagery: A window into the mechanisms and alterations of the motor system. Cortex; A Journal Devoted to the Study of the Nervous System and Behavior, 44(5), 494-506.

Decety, J., Jeannerod, M., Durozard, D., \& Baverel, G. (1993). Central activation of autonomic effectors during mental simulation of motor actions in man. Journal of Physiology, 461, 549-563.

Dolan, R. J., \& Fletcher, P. C. (1997). Dissociating prefrontal and hippocampal function in episodic memory encoding. Nature, 388(6642), 582-585.

Duvernoy, Henri M., Cabanis, E. A., \& Vannson, J. L. (1991). The human brain: Surface, three-dimensional sectional anatomy and MRI. Wien: Springer-Verlag.

Eickhoff, S. B., Stephan, K. E., Mohlberg, H., Grefkes, C., Fink, G. R., Amunts, K., et al (2005). A new SPM toolbox for combining probabilistic cytoarchitectonic maps and functional imaging data. Neuroimage, 25(4), 1325-1335.

Friston, K. J., Holmes, A. P., Worsley, K. J., Poline, J. B., Frith, C., \& Frackowiak, R. S. (1995). Statistical parametric maps in functional imaging: A general linear approach. Human Brain Mapping, 2, 189-210.

Friston, K. J., Holmes, A., Poline, J. B., Price, C. J., \& Frith, C. D. (1996). Detecting Activations in PET and fMRI: Levels of Inference and Power. Neuroimage, 4(3), 223-235.

Friston, K. J., Buechel, C., Fink, G. R., Morris, J., Rolls, E., \& Dolan, R. J. (1997). Psychophysiological and modulatory interactions in neuroimaging. Neuroimage, 6(3), 218-229.

Frith, C. D., Blakemore, S. J., \& Wolpert, D. M. (2000). Abnormalities in the awareness and control of action. Philosophical Transactions of the Royal Society of London B Biological Sciences, 355(1404), 1771-1788.
Frith, C. D., Moody, S. L., \& Driver, J. (2000). The role of dorsolateral prefrontal cortex in the selection of action control of cognitive processes: Attention and performance XVIII. Cambridge: MIT Press.

Fuster, J. M. (2000). Executive frontal functions. Experimental Brain Research, 133(1), 66-70.

Gaffan, D. (2005). Neuroscience. Widespread cortical networks underlie memory and attention. Science, 309(5744), 2172-2173.

Geyer, S., Schleicher, A., \& Zilles, K. (1999). Areas 3a, 3b, and 1 of human primary somatosensory cortex. Neuroimage, 10(1), 63-83.

Ghaffar, O., Staines D W. R., \& Feinstein, A. (2006). Unexplained neurologic symptoms: An fMRI study of sensory conversion disorder. Neurology, 67(11), 2036-2038.

Gilbert, S. J., Spengler, S., Simons, J. S., Steele, J. D., Lawrie, S. M., Frith, C. D., et al. (2006). Functional specialization within rostral prefrontal cortex (area 10): A meta-analysis. Journal of Cognitive Neuroscience, 18(6), 932-948.

Gitelman, Darren R., Penny, William D., Ashburner, J., \& Friston, Karl J. (2003). Modeling regional and psychophysiologic interactions in fMRI: The importance of hemodynamic deconvolution. Neuroimage, 19(1), 200-207.

Goldberg, I. I., Harel, M., \& Malach, R. (2006). When the brain loses its self: Prefrontal inactivation during sensorimotor processing. Neuron, 50(2), 329-339.

Graziano, M. S., Taylor, C. S., \& Moore, T. (2002). Complex movements evoked by microstimulation of precentral cortex. Neuron, 34(5), 841-851.

Grefkes, C., Geyer, S., Schormann, T., Roland, P., \& Zilles, K. (2001). Human somatosensory area 2: Observer-independent cytoarchitectonic mapping, interindividual variability, and population map. Neuroimage, 14(3), 617-631.

Grush, R. (2004). The emulation theory of representation: Motor control, imagery, and perception. The Behavioral and Brain Sciences, 27(3), 377-396.

Halligan, P. W., Athwal, B. S., Oakley, D. A., \& Frackowiak, R. S. J. (2000). Imaging hypnotic paralysis: Implications for conversion hysteria. The lancet, 355(9208), 986-987.

Halligan, Peter W., Bass, C., \& Marshall, John C. (2001). Contemporary approaches to the study of hysteria: Clinical and theoretical perspectives. USA: Oxford University Press.

Jahn, K., Deutschlander, A., Stephan, T., Strupp, M., Wiesmann, M., \& Brandt, T. (2004). Brain activation patterns during imagined stance and locomotion in functional magnetic resonance imaging. Neuroimage, 22(4), 1722-1731.

Jeannerod, M. (1994). The representing brain: Neural correlates of motor intention and imagery. Behavioral and Brain Science, 17, 187-245.

Kanaan, R. A. A., Craig, T. K. J., Wessely, S. C., \& David, A. S. (2007). Imaging repressed memories in motor conversion disorder. Psychosomatic Medicine, 69(2), 202-1202.

Liepert, J., Hassa, T., Tüscher, O., \& Schmidt, R. (2009). Abnormal motor excitability in patients with psychogenic paresis. Journal of Neurology, 256(1), $121-126$.

Mailis-Gagnon, A., Giannoylis, I., Downar, J., Kwan, C. L., Mikulis, D. J., Crawley, A. P., et al. (2003). Altered central somatosensory processing in chronic pain patients with "hysterical" anesthesia. Neurology, 60(9), 1501-1507.

Marshall, J. C., Halligan, P. W., Fink, G. R., Wade, D. T., \& Frackowiak, R. S. (1997). The functional anatomy of a hysterical paralysis. Cognition, 64(1), B1-B8.

Maruff, P., \& Velakoulis, D. (2000). The voluntary control of motor imagery. Imagined movements in individuals with feigned motor impairment and conversion disorder. Neuropsychologia, 38(9), 1251-1260.

Ochsner, K. N., \& Gross, J. J. (2005). The cognitive control of emotion. Trends in cognitive sciences, 9(5), 242-249.

Parsons, L. M. (1987). Imagined spatial transformation of one's body. Journal of Experimemenal Psychology General, 116(2), 172-191.

Parsons, L. M., Gabrieli, J. D., Phelps, E. A., \& Gazzaniga, M. S. (1998). Cerebrally lateralized mental representations of hand shape and movement. Journal of Neuroscience, 18(16), 6539-6548.

Passingham, R. E. (1993). The frontal lobes and voluntary action. Oxford: Oxford University Press.

Petersson, K. M., Nichols, T. E., Poline, J. B., \& Holmes, A. P. (1999). Statistical limitations in functional neuroimaging. II. Signal detection and statistical inference. Philosophical Transactions of the Royal Society of London B: Biological Sciences, 354, 1261-1281.

Petrides, M. (2005). Lateral prefrontal cortex: Architectonic and functional organization. Philosophical Transactions of the Royal Society of London B Biological Sciences, 360(1456), 781-795.

Price, C. J., \& Friston, K. J. (2002). Functional imaging studies of neuropsychological patients: Applications and limitations. Neurocase, 8(5), 345-354.

Rajkowska, G., \& Goldman-Rakic, P. S. (1995). Cytoarchitectonic definition of prefrontal areas in the normal human cortex: II. Variability in locations of areas 9 and 46 and relationship to the Talairach Coordinate System. Cerebral Cortex, 5, 323-337.

Roelofs, K., \& Spinhoven, P. (2007). Trauma and medically unexplained symptoms towards an integration of cognitive and neuro-biological accounts. Clinical Psychology Review, 27, 798-820.

Roelofs, K., Näring, G. W. B., Keijsers, G. P. J., Hoogduin, C. A. L., Van Galen, G. P., \& Maris, E. (2001). Motor imagery in conversion paralysis. Cognitive Neuropsychiatry, 6(1), 21-40.

Rossini, P. M., Caramia, D., Bassetti, M. A., Pasqualetti, P., Tecchio, F., \& Bernardi, G. (1996). Somatosensory evoked potentials during the ideation and execution of individual finger movements. Muscle Nerve, 19, 191-202.

Rowe, J., Friston, K., Frackowiak, R., \& Passingham, R. (2002). Attention to action: Specific modulation of corticocortical interactions in humans. Neuroimage, 17(2), 988-998. 
Rowe, J. B., Sakai, K., Lund, T. E., Ramsoy, T., Christensen, M. S., Baare, W. F., et al. (2007). Is the prefrontal cortex necessary for establishing cognitive sets? Journal of Neuroscience, 27(48), 13303-13310.

Schacter, D. L., \& Wagner, A. D. (1999). Medial temporal lobe activations in fMRI and PET studies of episodic encoding and retrieval. Hippocampus, 9(1), $7-24$.

Sekiyama, K. (1982). Kinesthetic aspects of mental representations in the identification of left and right hands. Perception and Psychophysics, 32(2), 89-95.

Shadmehr, R., \& Krakauer, J. W. (2008). A computational neuroanatomy for motor control. Experimental Brain Research, 185(3), 359-381.

Spence, S. A., Crimlisk, H. L., Cope, H., Ron, M. A., \& Grasby, P. M. (2000). Discrete neurophysiological correlates in prefrontal cortex during hysterical and feigned disorder of movement. The Lancet, 355(9211), 1243-1244.
Stone, J.,Zeman, A., Simonotto, E., Meyer, M., Azuma, R., Flett, S., et al. (2007). FMRI in patients with motor conversion symptoms and controls with simulated weakness. Psychosomatic Medicine, 69(9), 961-1961.

Summerfield, J. J., Hassabis, D., \& Maguire, E. A. (2009). Cortical midline involvement in autobiographical memory. Neurolmage, 44, 1188-1200.

Voss, M., Ingram, J. N., Wolpert, D. M., \& Haggard, P. (2008). Mere expectation to move causes attenuation of sensory signals. PLOS ONE, 3(8), e2866.

Vuilleumier, Patrik. (2005). Hysterical conversion and brain function. Progress in Brain Research, 150, 309-329.

Vuilleumier, P., Chicherio, C., Assal, F., Schwartz, S., Slosman, D., \& Landis, T. (2001) Functional neuroanatomical correlates of hysterical sensorimotor loss. Brain: $A$ Journal of Neurology, 124(Pt. 6), 1077-1090.

Wolpert, D. M., \& Ghahramani, Z. (2000). Computational principles of movement neuroscience. Nature Neuroscience, 3, 1212-1217. 